\title{
ADAPTACIÓN Y RENDIMIENTO DE LA VARIEDAD DE FRIJOL “NEGRO MEDELLÍN" EN EL SURESTE DE MÉXICO"
}

\author{
Ernesto López ${ }^{2}$, Javier Cumpían ${ }^{2}$, Enrique N. Becerra ${ }^{2}$, Bernardo Villar ${ }^{3}$, \\ Francisco J. Ugalde 2 , Jorge A. Acosta ${ }^{4}$
}

\begin{abstract}
RESUMEN
Adaptación y rendimiento de la variedad de frijol “Negro Medellín” en el sureste de México. El presente estudio se llevó a cabo de 1994 a 1997 con el objetivo de evaluar la adaptación y rendimiento de la nueva variedad de frijol "Negro Medellín” en la región del Trópico Húmedo del sureste de México. Se condujeron diez ensayos de rendimiento, cinco en el estado de Veracruz, cuatro en Chiapas y uno en Guerrero. Los ensayos incluyeron 16 genotipos y se realizaron, de acuerdo a las facilidades disponibles en los sitios de prueba, bajo condiciones de humedad residual o de temporal. En las evaluaciones se utilizó un diseño de Bloques Completos al Azar con tres repeticiones. La variedad Negro Medellín resultó sobresaliente por su rendimiento, amplia adaptación, con respuesta superior en ambientes favorables y consistente (1226 kg/ha, bi >1,0, $\left.\mathrm{S}^{2} \mathrm{di}=0\right)$. Durante las evaluaciones, la variedad Negro Medellín resultó tolerante a las enfermedades: virus del mosaico dorado (BGMV), mancha angular (Phaoisariopsis griseola) y roya (Uromyces appendiculatus var. appendiculatus), enfermedades de amplia distribución en la región tropical. Sin embargo, en una localidad de Veracruz Negro Medellín resultó susceptible a la antracnosis (Colletotrichum lindemuthianum). Además, la variedad Negro Medellín se validó en parcelas comerciales en campos de agricultores en diez localidades del sureste de México, localidades con clima tropical. En éstas parcelas comerciales, Negro Medellín resultó superior en rendimiento a diversos testigos, variedades criollas y mejoradas, de un 10 a $25 \%$, obteniendo un rendimiento promedio de $1300 \mathrm{~kg} / \mathrm{ha}$. La variedad Negro Medellín está en trámite para su registro como nueva variedad para las regiones productoras del sureste de México.
\end{abstract}

\begin{abstract}
Adaptation and yield of Negro Medellín bean in the southeast of Mexico. The present study was carried out from 1994 to 1997 with the aim of testing the adaptation and seed yield of the new bean cultivar "Negro Medellín" in the humid tropical region of Southeast Mexico. Five trials were conducted in the state Veracruz, four in Chiapas and one in Guerrero. Trials were conducted according to available facilities at each site under residual moisture or rainfall conditions. Trials included 16 bean genotypes that were distributed in the field using a Complete Random Design with three replicates. Negro Medellín resulted outstanding in average yield, yield stability and wide adaptation, although it showed a better response in favorable environments (1226 $\mathrm{kg} / \mathrm{ha}$, bi $>1$ and $\mathrm{S}^{2} \mathrm{~d}=0$ ). During the conduction of the trials, Negro Medellín was tolerant to widespread diseases: bean golden mosaic virus (BGMV), angular leaf spot (Phaoisariopsis griseola) and rust (Uromyces appendiculatus var. appendiculatus). However, in a location in the state of Veracruz, Negro Medellín was severely attacked by anthacnose (Colletotrichum lindemuthianum). In addition, Negro Medellín was evaluated in commercial plots in farmer's fields at 10 locations in the humid tropics of southeast Mexico. In those plots Negro Medellín was compared to a set of different checks that included bred cultivars and landraces. Negro Medellín outyielded all checks by 10 to $25 \%$ and its average yield was $1300 \mathrm{~kg} / \mathrm{ha}$. Negro Medellín is in the process of being registered as a new bred bean cultivar for the lowland humid tropics of southeast Mexico.
\end{abstract}

\section{INTRODUCCIÓN}

Los principales estados productores del frijol en el sureste de México son Chiapas y Veracruz, con 110 y $50 \mathrm{mil}$ ha. anuales, respectivamente. Lo anterior repre- senta el $90 \%$ de la superficie cultivada de esta leguminosa en la región tropical de México (SAGAR, 1997). Los problemas bióticos y abióticos que enfrenta el cultivo del frijol en ambas entidades son similares a los encontrados en el resto de los estados del sur de México.

\footnotetext{
1 Presentado en la XLVI Reunión Anual del PCCMCA, Puerto Rico, 2000.

2 Programa de frijol CECOT-CIRGOC- INIFAP, Apdo. Postal No. 429 Veracruz, Ver. , México.

3 Programa Frijol CECCH- CIRPAS-INIFAP. Apdo. Postal No. 1 Ocozocoautla, Chis., México.

${ }^{4}$ Programa de Frijol INIFAP. Apdo. Postal No. 10, Chapingo, Mex. CP 56230
} 
Entre los factores bióticos adversos sobresalen: la mosca blanca (Bemicia tabaci), la doradilla (Diabrótica balteata) y la chicharrita (Empoasca kraemeri), el virus del mosaico dorado (BGMV), la roya (Uromyces appendiculatus var. appendiculatus), y la mancha angular (Phaoisariopsis griseola); entre los factores abióticos adversos sobresalen la sequía terminal, la presencia de suelos ácidos y suelos de baja fertilidad (López y Acosta, 1998).

El frijol negro es la clase de mayor demanda a nivel nacional y uno de los tipos de grano que más se importa; se estima que se consumen alrededor de $450 \mathrm{mil}$ toneladas al año en el sur y centro del país (Castellanos et al., 1997)

En los últimos años, en el desarrollo de las variedades mejoradas para la región del sureste de México por el programa de frijol del Campo Cotaxtla, del INIFAP, se han utilizado las variables de estabilidad, con base en el modelo propuesto por Eberhart y Russell (1966) para definir la amplitud de adaptación de las mismas. En 1981, se liberó la variedad Negro Huasteco-81. Los resultados obtenidos en cinco estados del sur de México señalaron a esta variedad como estable, tolerante al virus del mosaico dorado y resistente a la roya, así como con alto potencial de rendimiento (Yoshii y Rodríguez, 1981). En 1992 se liberó la variedad Negro INIFAP para el estado de Chiapas y regiones tropicales similares. Esta variedad se evaluó como línea mejorada en ocho localidades de la región central de Chiapas y de acuerdo a la calificación propuesta por Carballo y Márquez (1970) se reportó como variedad estable (Villar y López, 1994). En evaluaciones posteriores en seis ambientes del trópico húmedo de México, esta variedad mostró amplia adaptación, estabilidad y alto rendimiento (López et al., 1994). En 1993 se liberó para la costa de Chiapas la variedad Negro Tacaná (Fraire, 1993). Esta variedad y la línea II-307 que dio origen a la variedad Negro Medellín que aquí se describe, fueron evaluadas en el sureste de México, durante tres ciclos agrícolas de otoño-invierno de 1992 a 1994. Estas variedades mostraron altos rendimientos, amplia adaptación y estabilidad, (López et al., 1996), respon- diendo mejor en ambientes favorables en forma consistente (López et al., 1997). El objetivo del presente estudio fue determinar la adaptación y estabilidad de rendimiento de la nueva variedad de frijol Negro Medellín en la región del trópico húmedo del sureste de México.

\section{MATERIALES Y MÉTODOS}

\section{Evaluación del rendimiento y adaptación de la va- riedad Negro Medellín}

Durante el periodo 1994-1997, se condujeron diez ensayos uniformes de rendimiento, bajo condiciones de humedad residual y temporal; cinco en el estado de Veracruz, cuatro en Chiapas y uno en Guerrero. Las principales características de los sitios de prueba se presentan en el Cuadro 1. En los ensayos, entre 16 genotipos se incluyó la variedad Negro Medellín y los testigos Negro Jamapa y Negro Tacaná, que son las principales variedades recomendadas para la zona tropical húmeda de México. En todos los ensayos se utilizó un diseño de Bloques Completos al Azar con tres repeticiones y parcelas de tres surcos de cinco $m$ de longitud. La fecha de siembra de los experimentos fue de septiembre a octubre. Los ensayos se condujeron siguiendo las recomendaciones agronómicas para cada localidad.

En todas las localidades se tomaron datos sobre reacción a las enfermedades que ocurrieron durante el ciclo, la fenología de las variedades y el rendimiento. El rendimiento de grano se calculo en $\mathrm{kg} / \mathrm{ha}$ al $14 \%$ de humedad. Los datos del rendimiento se sometieron a un análisis de varianza individual y después se realizó un análisis combinado de los diez experimentos. Para comparar las medias de tratamiento se utilizó la prueba de Tukey a un nivel de significancia del 0,05\%. También se realizó un análisis de regresión con los diez experimentos por el método de Eberhart y Russell (1966), y la adaptación y estabilidad de las variedades se calificaron con base al coeficiente de regresión y la desviación de la regresión (Carballo y Marquez, 1970).

Cuadro 1. Localización geográfica, temperatura y precipitación media anual en las localidades donde se realizaron los ensayos de rendimiento en la región del sureste de México.

\begin{tabular}{lccccc}
\hline \multicolumn{1}{c}{ Localidad } & Latitud & Longitud & Altitud (msnm) & Temperatura ${ }^{\circ} \mathbf{C}$ & Precipitación (mm) \\
\hline Chiapas-Sur & $14^{\circ} 15^{\prime}$, & $92^{\circ} 15^{\prime}$ & 137 & 26,0 & 2488 \\
Chiapas-Centro & $16^{\circ} 46^{\prime}$ & $93^{\circ} 27^{\prime}$ & 846 & 23,6 & 898 \\
Guerrero-Centro & $17^{\circ} 19^{\prime}$ & $98^{\circ} 58^{\prime}$ & 1420 & 20,0 & 1200 \\
Veracruz-Sur & $18^{\circ} 06^{\prime}$ & $95^{\circ} 53^{\prime}$ & 25 & 25,0 & 1762 \\
Veracruz-Centro & $19^{\circ} 12^{\prime}$ & $96^{\circ} 81^{\prime}$ & 16 & 25,5 & 1668 \\
Veracruz-Norte & $20^{\circ} 57^{\prime}$ & $97^{\circ} 24^{\prime}$ & 14 & 24,2 & 1351 \\
\hline
\end{tabular}




\section{Evaluación de la variedad Negro Medellín en parce- las de validación}

La variedad Negro Medellín se evaluó en parcelas comerciales en diez localidades del sureste de México durante el ciclo agrícola de 1998-99,y se comparó con variedades locales y variedades mejoradas. Las siembras se realizaron al tiempo que se establecieron lotes comerciales en cada localidad durante los meses de septiembre y octubre. La superficie por lote de validación fue variable en cada sitio de acuerdo con la disponibilidad de semilla y terreno. El rendimiento se calculó en $\mathrm{kg} / \mathrm{ha}$ en base al área total sembrada. Estas parcelas se establecieron en terrenos de agricultores cooperantes, los que se encargaron de la conducción agronómica y cosecha de las mismas.

\section{Evaluación de reacción a enfermedades}

Durante la conducción del ensayo uniforme de 1994 a 1997, se calificó la reacción de los genotipos evaluados a la incidencia del virus del mosaico dorado y la mancha angular en el centro y sur de Chiapas, con una escala de 1 a 9 (Shoonhoven y Pastor-Corrales, 1987), cuyos valores son: 1 a $2=$ resistente, 3 a $4=$ tolerante, 5 a $6=$ medianamente tolerante, 7 a $8=$ medianamente susceptible y $9=$ susceptible. También se calificó la reacción a la roya, con una escala de 1 a 5 , según la cual $1=$ inmune; $2=$ resistente $3=$ moderadamente resistente; $4=$ moderadamente susceptible y $5=$ susceptible. La reacción a las enfermedades se realizó durante la etapa reproductiva.

\section{Estudios de la calidad tecnológica del grano}

La calidad tecnológica y nutricional de la variedad Negro Medellín y de los genotipos Negro Michigan (T39) y DOR-500 se evaluó en el laboratorio de calidad del programa nacional de frijol del INIFAP en Texcoco, Edo. de México. Los materiales para esta evaluación se cosecharon el 14 de enero de 1999. Las determinaciones de peso y tamaño de grano, porcentaje de testa, absorción de agua, tiempo de cocción e índice de espesor de caldo, se hicieron de acuerdo a las técnicas del Instituto de Nutrición de Centroamérica y Panamá, INCAP (Elías et al., 1986). El tiempo de cocción se determinó por el método sensorial. El análisis químico del grano se efectuó sobre una muestra molida y se evaluó el contenido de proteína por el método de Kjeldhal. No se realizó análisis estadístico con la información generada y los resultados que se presentan son promedio de tres valores.

\section{RESULTADOS Y DISCUSIÓN}

\section{Evaluación del rendimiento y adaptación de la va- riedad Negro Medellín.}

En la mayoría de los sitios de prueba existieron diferencias altamente significativas entre variedades y en el análisis conjunto hubo diferencias significativas entre variedades, localidades y para la interacción variedad por localidad. Los genotipos que mostraron los mejores rendimientos en el análisis conjunto fueron Negro Medellín, DOR-500, II-68-FGCO-29 y Negro Tacaná, los que resultaron estadísticamente iguales entre sí y con la variedad comercial Negro Cotaxtla-91, y superiores a Negro Jamapa. Las localidades en donde se obtuvieron los mayores rendimientos fueron: Zitlala, Guerrero y Tihuatlán, Ver., mientras que los rendimientos más bajos se obtuvieron en Ocozocoautla, Chis., en los ciclos OI- 95-96 y 97-98. Los bajos rendimientos en esta última localidad fueron debidos a un severo ataque del virus del mosaico dorado y a la falta de humedad en las etapas reproductivas del cultivo, respectivamente (Cuadro 2).

Cuadro 2. Promedio de rendimiento de 16 genotipos de frijol en 10 ambientes del trópico húmedo de México. Periodo1994-1997

\begin{tabular}{lllc}
\hline \multicolumn{1}{c}{ Localidad } & Ciclo y año & \multicolumn{1}{c}{ Condición } & Rendimiento kg/ha \\
\hline Zitlala, Guerrero & PV-1997 & Temporal & $1417 \mathrm{a}^{1}$ \\
Tihuatlán, Ver. & OI-1994-95 & Humedad Residual & $1366 \mathrm{ab}$ \\
Tapachula, Chiapas & OI-1996-97 & Humedad Residual & $1270 \mathrm{~b}$ \\
Isla, Veracruz & OI-1996-97 & Humedad Residual & $1035 \mathrm{c}$ \\
Tapachula, Chiapas & OI-1997-98 & Humedad Residual & $976 \mathrm{c}$ \\
Isla, Veracruz & OI-1995-96 & Humedad Residual & $941 \mathrm{c}$ \\
Isla, Veracruz & OI-1994-95 & Humedad Residual & $937 \mathrm{~cd}$ \\
Cecot, Veracruz & OI-1996-97 & Humedad Residual & $806 \mathrm{de}$ \\
Ocozocoautla, Chiapas & OI-1995-96 & Humedad Residual & $752 \mathrm{e}$ \\
Ocozocoautla, Chiapas & OI-1997-98 & Humedad Residual & $568 \mathrm{f}$ \\
\hline
\end{tabular}

1. Prueba de Tukey 0,05 
Los rendimientos promedio de diez ambientes, los valores de los parámetros bi y $S^{2}$ di y la clasificación de cada una de los genotipos con base en el esquema de calificación propuesto por Carballo y Marquez (1970), se presentan en el Cuadro 3. De los 16 genotipos evaluados para rendimiento en los diez ambientes, 11 resultaron estables en su respuesta $\left(b i=0 ; S^{2} d i=0\right)$; dentro de esta categoría se encontraron las variedades Negro INIFAP y Negro Jamapa, junto con nueve líneas experimentales. Tres genotipos mostraron buena respuesta en todos los ambientes pero resultaron inconsistentes (bi = $0, S^{2}$ di > 0): DOR 500, Negro Tacaná y II-283-CB-5EOE-M. La variedad Negro Medellín respondió mejor en ambientes favorables en forma consistente ( bi >1; $\mathrm{S}^{2} \mathrm{di}$ $=$ O). Por último, la línea 1708, se adaptó mejor en ambientes desfavorables y fue consistente $\left(\mathrm{bi}<1 ; \mathrm{S}^{2} \mathrm{di}=\right.$ O). Los resultados anteriores difieren con los reportados por López et al. (1999), en donde las variedades Negro Medellín y Negro Tacaná mostraron calificaciones diferentes a las de este trabajo, mientras que los resultados reportados con las variedades comerciales Jamapa y Negro INIFAP fueron similares.

\section{Evaluación de la variedad Negro Medellín en parce- las de validación}

Las condiciones agroclimáticas ocurridas durante el ciclo agrícola de evaluación (datos no disponibles) fueron favorables para el cultivo del frijol. En la evaluación de la variedad Negro Medellín en diez parcelas de validación establecidas en Veracruz y Chiapas se obtuvo un rendimiento medio de $1300 \mathrm{~kg} / \mathrm{ha}$ (Cuadro 4). Los rendimientos obtenidos a través de las localidades de validación fueron superiores a los rendimientos de los testigos utilizados, variedades criollas y mejoradas. La variedad Negro Medellín resultó sobresaliente por su alto potencial de rendimiento, amplia adaptación y tolerancia a las enfermedades que ocurren en la región tropical de México (virus del mosaico dorado, roya y mancha angular), así como con adaptación a los suelos de baja fertilidad y ácidos.

Cuadro 3. Rendimiento promedio y parámetros de estabilidad de 16 líneas de frijol negro en 10 localidades del sureste de México

\begin{tabular}{lccrc}
\hline Genotipo & kg/ha & bi & S2di & Clasificación \\
\hline II-307-CB-5E-OE-M-M-M & 1226 & 1,53 & -8965 & $\mathrm{E}$ \\
DOR-500 & 1189 & 0,67 & 72479 & $\mathrm{~B}$ \\
II-68-F60C-29 & 1070 & 1,43 & -6114 & $\mathrm{~A}$ \\
Negro Tacaná & 1062 & 0,5 & 130.663 & $\mathrm{~B}$ \\
1713 & 1049 & 1,49 & 6706 & $\mathrm{~A}$ \\
II-283-CB-5E-0E & 1046 & 0,67 & 70.136 & $\mathrm{~B}$ \\
1467 & 1043 & 1,44 & 12.052 & $\mathrm{~A}$ \\
1670 & 1016 & 1,21 & 12.637 & $\mathrm{~A}$ \\
1684 & 1013 & 1,04 & -14.950 & $\mathrm{~A}$ \\
1671 & 1000 & 1,28 & -20.502 & $\mathrm{~A}$ \\
Negro INIFAP & 993 & 1,20 & -21.185 & $\mathrm{~A}$ \\
SM-98 & 948 & 1,08 & -3951 & $\mathrm{~A}$ \\
1708 & 933 & 0,61 & -23.587 & $\mathrm{C}$ \\
DOR-446 & 928 & 0,47 & 9.130 & $\mathrm{~A}$ \\
SM-52 & 908 & 0,96 & -16.319 & $\mathrm{~A}$ \\
Jamapa & 686 & 0,42 & 29.194 & $\mathrm{~A}$ \\
Promedio & 1007 & & & \\
\hline
\end{tabular}

$\mathrm{A}=$ estable

$\mathrm{B}=$ buena respuesta en todos los ambientes inconsistente.

$\mathrm{C}=$ responde mejor en ambientes desfavorables consistente

$\mathrm{E}=$ responde mejor en buenos ambientes consistente

\section{Evaluación de reacción a enfermedades}

La enfermedad del virus del mosaico dorado se evaluó en el centro de Chiapas (Ocozocoautla) en los ciclos de Otoño-Invierno 95-96 y 98-99; en este último también se calificó la reacción a la mancha angular. En el sur de Chiapas (Tuxtla Chico) se calificó la respuesta a un fuerte ataque del BGMV en el ciclo Otoño-Invierno 92-93. La variedad Negro Medellín mostró tolerancia al BGMV y a la mancha angular en el centro y sur de Chiapas. Confirmando la resistencia previamente reportada por López et al. (1999) y López et al. (1994).

Cuadro 4. Rendimiento promedio en $\mathrm{kg} / \mathrm{ha}$ de la variedad Negro Medellín en parcelas de validación en el sureste de México.

\begin{tabular}{lcccl}
\hline \multicolumn{1}{c}{ Localidad } & Ciclo & Negro Medellín & kg/ha & \multicolumn{1}{c}{ Testigo } \\
\hline Veracruz, Veracruz & OI-98-99 & 1597 & 762 & Negro Criollo \\
Veracruz, Veracruz & OI-98-99 & 838 & 580 & Jamapa \\
Jamapa, Veracruz & OI-98-99 & 2105 & 1000 & Negro Bolita \\
M. de Bravo, Veracruz & OI-97-98 & 1661 & - & Jamapa \\
M. de Bravo, Veracruz & OI-98-99 & 864 & 600 & Negro INIFAP \\
Ocozocuatla, Chiapas & OI-98-99 & 673 & 762 & Negro Michigan \\
Ixtacuaco, Veracruz & OI-98-99 & 1539 & 1171 & Jamapa \\
Isla, Veracruz & OI-98-99 & 959 & 620 & Negro Regional \\
San Andres Tuxtla, Veracruz & OI-98-99 & 1756 & 600 & Negro Tacaná \\
Isla, Veacruz. & OI-98-99 & 1010 & 931 & \\
Promedio & & 1300 & & \\
\hline
\end{tabular}


Cuadro 5. Calidad tecnológica y nutricional del grano de la variedad Negro Medellín, en relación con otras dos variedades mejoradas.

\begin{tabular}{|c|c|c|c|c|c|c|c|c|}
\hline Variedad & $\begin{array}{c}\text { Humedad } \\
\%\end{array}$ & $\begin{array}{c}\text { Peso } 100 \\
\text { granos }(\mathrm{g})\end{array}$ & $\begin{array}{c}\text { Vol.100 } \\
\text { granos (mil.) }\end{array}$ & $\begin{array}{c}\text { Testa } \\
\%\end{array}$ & $\begin{array}{c}\text { Absn. de agua a } \\
18 \text { h. de remojo } 1(\%)\end{array}$ & $\begin{array}{l}\text { Tiempo de cocción } \\
\text { minutos }\end{array}$ & $\begin{array}{c}\text { Sólidos } \\
\text { en caldo }(\%)\end{array}$ & $\begin{array}{c}\text { Proteina }^{1} \\
\%\end{array}$ \\
\hline N. Medellín & 12,63 & 25,7 & 16 & 8,64 & 94,7 & 80 & 0,26 & 22,1 \\
\hline DOR-500 & 12,67 & 24,4 & 18 & 9,09 & 89,3 & 94 & 0,26 & 20,9 \\
\hline $\mathrm{N}$, Michigan & 11,58 & 20,34 & 16 & 8,75 & 103,3 & 99 & 0,26 & 20,0 \\
\hline
\end{tabular}

${ }^{1}$ En base a peso seco.

La reacción a la roya se calificó en el centro de Veracruz, en los ciclos de Primavera- Verano 94-94 y 9797 y en el norte de Veracruz, en el ciclo Primavera-Verano 93-93. Los resultados indicaron que Negro Medellín resultó resistente, en contraste con la variedad Jamapa que resultó susceptible .Los resultados en la reacción a la roya fueron similares a los reportados por López et al. (1999), López et al. (1994).

\section{Estudios de la calidad tecnológica del grano}

Los resultados de la calidad tecnológica (Cuadro 5) mostraron que la variedad Negro Medellín posee características físicas diferentes a las variedades incluidas en la evaluación en peso de grano, tamaño y porcentaje de testa. El tiempo de cocción de Negro Medellín fue menor que los otros dos genotipos estudiados en 14 y 19 minutos, respectivamente. Las tres variedades presentaron un espesor del caldo de cocción muy similar. En el aspecto de calidad nutricional, Negro Medellín al igual que los otros genotipos tuvieron un contenido proteínico dentro del promedio para frijol negro tropical, superior al 20\% en base seca (Hernández et al., 1984; Jacinto et al., 1993).

\section{CONCLUSIONES}

Se identificaron 11 genótipos de frijol estables en rendimiento a través de las localidades de prueba.

Las localidades de mayor potencial de rendimiento fueron: Zitlala en Guerrero y Tihuatlán, en Veracruz con un rendimiento medio aproximado a $1400 \mathrm{~kg} / \mathrm{ha}$.

Los genotipos que mostraron los mayores rendimientos fueron: Negro Medellín, DOR-500, II-68FGOC-29 y Negro Tacaná, con rendimientos promedio de diez ensayos de hasta $1226 \mathrm{~kg} / \mathrm{ha}$. El menor rendimiento promedio se observó con el testigo Negro Jamapa con $686 \mathrm{~kg} / \mathrm{ha}$.
La variedad Negro Medellín resultó sobresaliente por su rendimiento, amplia adaptación, con respuesta superior en ambientes favorables y consistente (1226 $\mathrm{kg} / \mathrm{ha}$, bi $\left.>1,0, \mathrm{~S}^{2} \mathrm{di}=0\right)$. Con excepción de la antracnosis, la variedad Negro Medellín resultó tolerante a las enfermedades.

\section{LITERATURA CITADA}

CARBALlO, C. A.; F. MARQUEZ, S. 1970. Comparación de variedades de maíz del bajío y la mesa central por su rendimiento y estabilidad. Agrociencia 5: 129-146.

SCHOONCHOVEN A.; PASTOR-CORRALES, M.A. 1987. Sistema estándar para la evaluación de germoplasma de frijol. Centro Internacional de Agricultura Tropical. Cali, Colombia. p. 56

CASTELLANOS, R.J.; GUZMÁN, H.; JIMENÉZ, A.; MEJÍA,C.; MUÑOZ, J.J.; ACOSTA, J.; HOYOS, G.; LÓPEZ, E.; GONZÁLEZ, D.; SALINAS, R.; GONZÁLEZ, J.; MUÑOZ, J.; FERNÁNDEZ, P.; CERESA, C. 1997. Hábitos preferenciales de los consumidores de frijol común en México. Archivos Latinoamericanos de Nutrición 47(1):163-167

EBERHART, S. A.; W. A. RUSSELL. 1969. Stability parameters for comparing varieties, Crop Sci. $6: 36-40$

LÓPEZ, S. E.; VILLAR S, B.; CANO R, O. 1994. Rendimiento y adaptación del frijol Negro E-44 en el trópico Húmedo de México. Fitotecnia Mexicana Vol. 17: 39-47

LÓPEZ, S. E.; BECERRA, L. N. E.; CANO, R. O.; FRAIRE, V.G., 1994. Reacción al virus del mosaico dorado, adaptación y rendimiento de la línea DOR 390 en el sureste de México. Revista Mexicana de Fitopatología $12: 139-145$

LÓPEZ, S. E.; BECERRA, L. N. E.; CANO, R. O.; ORTEGA, Z. A. D.; ACOSTA, G. A. J. 1996. Adaptación y calidad tecnológica de la variedad de frijol Negro Tacaná. Agronomía Mesoamericana 7 (1): 26-34.

LÓPEZ S.E.; J.A. ACOSTA-GALLEGOS. 1998. Mejoramiento de frijol negro mesoamericano en el trópico de 
México. In: Memorias del Taller Internacional de Mejoramiento Genético de Frijol negro Mesoamericano. Veracruz, México, Programa Cooperativo Regional de Frijol para Centroamérica, México y el Caribe (PROFRIJOL). pp. 10-16,

ELÍAS, L.; GARCÍA, S.; R. BRESSANI. 1986. Métodos para establecer calidad tecnológica y nutricional de frijol (Phaseolus vulgaris L.) Instituto de Nutrición de Centroamérica y Panamá (INCAP). 41p.

HERNÁNDEZ, M.; CHÁVEZ, A.; BOURGES, H. 1984. Valor nutritivo de alimentos mexicanos. Tablas de uso práctico. Instituto Nacional de la Nutrición. Publicación de la división de nutrición. L-12. 34 p.
JACINTO, H. C.; ACOSTA, J.A.; ORTEGA, A. J. 1993. Caracterización del grano de variedades mejoradas de frijol en México. Agric. Tec. Mex. 19:167-179

SAGAR. 1997. FRIJOL. Estimación de cosecha de primavera-verano 1997. Documento de circulación interna. Subsecretaria de Agricultura y Ganadería. Dirección General de Agricultura y Ganadería, México, D.F. p.15

VILLAR, B.; E. LÓPEZ. 1993. Negro INIFAP, nueva variedad de frijol para Chiapas y regiones tropicales similares. Fitotecnia Mexicana 16(2):208-209.

YOSHII, O.K.; R. RODRÍGUEZ. 1982. Negro Huasteco 81, nueva variedad de frijol para el trópico de México. CECOT-CIRGOC-INIA-SARH, Folleto Técnico No. 1, p. 11. 